\title{
Improved Current Efficiency in Organic Light- Emitting Devices with Co-Doped Host Layer
}

\author{
Lishuang $\mathrm{Wu}$, Dandan Chen and Huishan Yang* \\ College of Physics and Information Engineering, Key Laboratory of Information Functional Material for Fujian Higher Education, \\ Quanzhou Normal University, Quanzhou 362000, People's Republic of China \\ ${ }^{*}$ Corresponding author
}

\begin{abstract}
A co-doping host layer 4,4'-N,N'-dicarbazolebiphenyl (CBP) and tris-(8-hydroxyqunoline)aluminum(Alq), was incorporated tris (2-Phenylpyridine) iridium [ $\operatorname{Ir}(\mathrm{ppy}) 3]$ doped into host as the emitting layer (EML) based organic lightemitting device. Such a structure helps to increase the energy transfer from host to guest resulting in an improved current efficiency. The highest luminous efficiency of the device with 6:4 of CBP: Alq achieved $11.9 \mathrm{~cd} / \mathrm{A}$, which is nearly 4.3 times that of the device with 8:2 of CBP: Alq, and the power efficiency achieved $4.69 \mathrm{~lm} / \mathrm{W}$, which is nearly 6.7 times that of the device with 8:2 of CBP: Alq.
\end{abstract}

Keywords — organic light emitting devices; co-doping host layer; current efficiency; power efficiency

\section{INTRODUCTION}

Organic light-emitting devices (OLEDs) would be probably promising ways to offer the satisfying new display technologies. In the past decade, the architecture of the OLEDs was studied for better power efficiency and device lifetime [113]. These emitting layer (EML) structures can be categorized as the uniformly mixed (UM) layer [8,9], step-wise graded mixed (SGM) layer [12] and continuously graded mixed (CGM) layer $[9,11,12]$. The common feature between these EMLs is that these are basically bipolar transport layers. The main difference between these three EML structures lies in the spatial distribution of the electron-and hole-transport materials. The EML of UM-OLED consists of a constant mixed composition, which is also known as the mixed host $(\mathrm{MH})$ OLED [13]. For SGM-OLED, the EML consists of multilayers with different holes transport material (HTM): electron transport material (ETM) compositions or ratios, hence it is coiled as a graded layer. For example, when Tris-(8hydroxyquinoline)aluminum(Alq3) is utilized as the ETM and EML, mixing it with HTM such as N,N'-Di-[(1-naphthyl)$\mathrm{N}, \mathrm{N}^{\prime}$-diphenyl]-1,1'-biphenyl)-4,4'-diamine(NPD), improves the stability of the OLED by limiting the formation of fluorescence quenching cationic Alq3 [14]. The electron and hole transport are gradually slowed down in the graded region due to the increasing hopping distance for electrons and holes. In this paper, we select CBP:Alq co-doping layer as the host, this design improves guest-host balance of energy transfer at the EML. Tuning of the current efficiency is possible by varying the ratio of host. To maintain the same optical out coupling, the total thickness of the organic layers was kept constant in our experiment. The highest luminous efficiency of the device with $6: 4$ of CBP: Alq achieved $11.9 \mathrm{~cd} / \mathrm{A}$, which is nearly 4 times that of the device with 8:2 of CBP: Alq, and the power efficiency achieved $4.69 \mathrm{~lm} / \mathrm{W}$, which is nearly 6.7 times that of the device with 8:2 of CBP: Alq, respectively.

\section{EXPERIMENTAL DETAILS}

Figure I shows the molecular structure of the materials used in this work, the structure of device. The structure of the device is ITO/m-MTDATA $(40 \mathrm{~nm}) / \mathrm{NPB}(10 \mathrm{~nm}) / \mathrm{CBP} \mathrm{x} \%$ : Alq y\%:Ir(ppy3) 8\% (20 nm)/ BPhen $(50 \mathrm{~nm}) / \mathrm{LiF}(1 \mathrm{~nm}) / \mathrm{Al}$ $(150 \mathrm{~nm})$ were fabricated, where $\mathrm{x}: \mathrm{y}=8: 2,6: 4,4: 6,2: 8$ and the corresponding devices are named $\mathrm{A}, \mathrm{B}, \mathrm{C}$ and $\mathrm{D}$, respectively. In our devices, 4, 4',4',- tris $\{\mathrm{N},-$ (3-methylphenyl)- $\mathrm{N}$ phenylamin triphenylamine (m-MTDATA) and N, N'- di (naphthalene-1-yl)- N,N'-diphenyl-benzidine (NPB) are used as hole-injecting layer and hole transport layer; 4,4'-N,N'dicarbazole-biphenyl (CBP) and tris-(8hydroxyqunoline)aluminum (Alq) acts as host; tris (2Phenylpyridine) iridium [Ir(ppy)3] was selected as the green guest; 4,7-diphenyl-1,10- phenanthroline (BPhen) acts as the electron transporting layer; $\mathrm{LiF}$ as an electron injecting layer, and $\mathrm{Al}$ as cathode electrode.

The routine cleaning procedure, including ultrasonication in acetone, Decon90, and rinsing in deionized water, was firstly carried out to clean ITO glass. The deposition was carried out in a high vacuum condition of about $5 \times 10-4 \mathrm{~Pa}$. Except for host materials, all other materials were deposited simultaneously for the four samples (the same evaporation process). This avoids the uncertainties in comparing devices fabricated with different evaporation processes. Electroluminescence (EL) spectra of the fabricated devices were measured with a PR655 Spectra Scan spectrometer. The luminance-current density-voltage characteristics were recorded simultaneously with the measurement of the EL spectra by combining the spectrometer with a Keithley model 2400 programmable voltage-current source. All measurements were carried out at room temperature under ambient atmosphere without any encapsulation of the OLEDs. 


\begin{tabular}{|c|}
\hline LiF $(1 \mathrm{~nm}) / \mathrm{Al}(200 \mathrm{~nm})$ \\
\hline BPhen $(50 \mathrm{~nm})$ \\
\hline \hline CBP x\%:Alq $\mathrm{y} \%: 8 \%$ Ir(ppy) $3(20 \mathrm{~nm})$ \\
\hline NPB $(10 \mathrm{~nm})$ \\
\hline m-MTDATA $(40 \mathrm{~nm})$ \\
\hline ITO \\
\hline
\end{tabular}
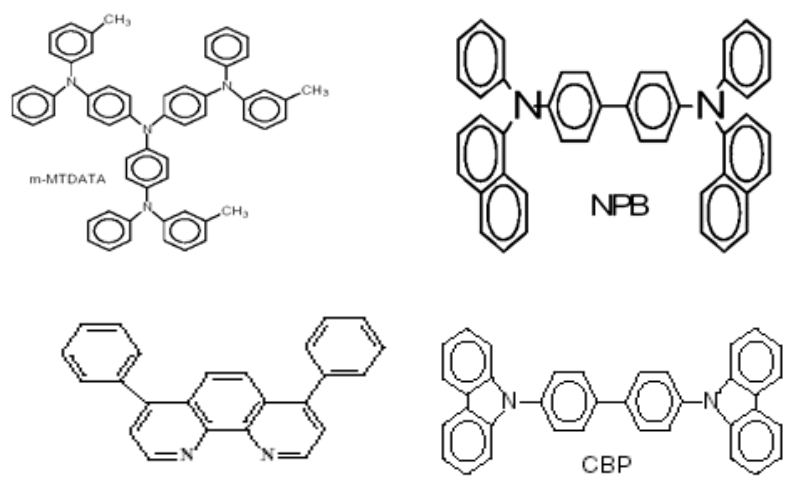

BPhen

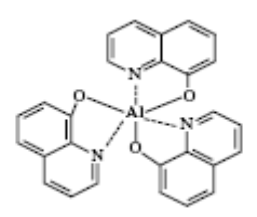

Alq

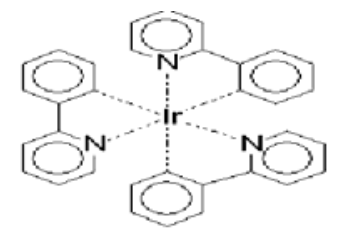

$\operatorname{Ir}(\mathrm{ppy})_{3}$

FIGURE I. THE DEVICE STRUCTURE AND CHEMICAL STRUCTURE OF ORGANIC MATERIALS OF THE OLEDS

\section{RESULTS AND DISCUSSION}

Four devices with different ratios of the co-doped host layer were fabricated and tested for this study. Figure 2(a) and Figure 2(b) shows the current density-voltage characteristics and the luminance- voltage characteristics of the devices. The current density of the device is determined mainly by the interfacial energy barrier and charge mobility of the organic materials. A low interfacial energy barrier and high carrier mobility usually give low driving voltage. In particular, interfacial energy barrier influences not only on the driving voltage but also the turn-on voltage of the devices. In other words, the turn-on voltage is closely related to the interfacial properties of the device, while the driving voltage mainly depends on the charge mobility of the material. It is essential to confine the electrons and holes in the light-emitting layer to get high performance. Under the forward bias conditions, the current increased super linearly with increasing applied voltage. It is found that the current density of device A-D at $4 \mathrm{~V}$ achieves $0.4711 \mathrm{~mA} / \mathrm{cm} 2$, $0.5275 \mathrm{~mA} / \mathrm{cm} 2, \quad 0.7045 \mathrm{~mA} / \mathrm{cm} 2$, and $6.1288 \mathrm{~mA} / \mathrm{cm} 2$, respectively. The luminance of the devices also showed the

same tendency as the current density. The luminance of the device A-D achieves $4.41 \mathrm{~cd} / \mathrm{m} 2,3.90 \mathrm{~cd} / \mathrm{m} 2,2.30 \mathrm{~cd} / \mathrm{m} 2$, and $21.88 \mathrm{~cd} / \mathrm{m} 2$, respectively. Due to the Alq can blocking hole and more exciton confinement within the EML. It is thought that accumulation of holes occurs at the EML. Therefore, the electric field of EML of OLED with the co-doped host layer is considered to be enhanced compared with the OLED without ones, thus the EL properties are improved. It is also considered that the improvement occurs by a good balance between the injected electrons and holes as Alq owns high electron mobility. It's well known that co-doped host layer confine holes and electrons effectively in the EML. The improvement seems to be caused by a good balance between the injected electrons and holes when the co-doped host layer with proper doping ratio. The luminance of devices A-D is $4089 \mathrm{~cd} / \mathrm{m} 2,10950 \mathrm{~cd} / \mathrm{m} 2$, $15390 \mathrm{~cd} / \mathrm{m} 2$ and $16370 \mathrm{~cd} / \mathrm{m} 2$ at $15 \mathrm{~V}$, respectively.

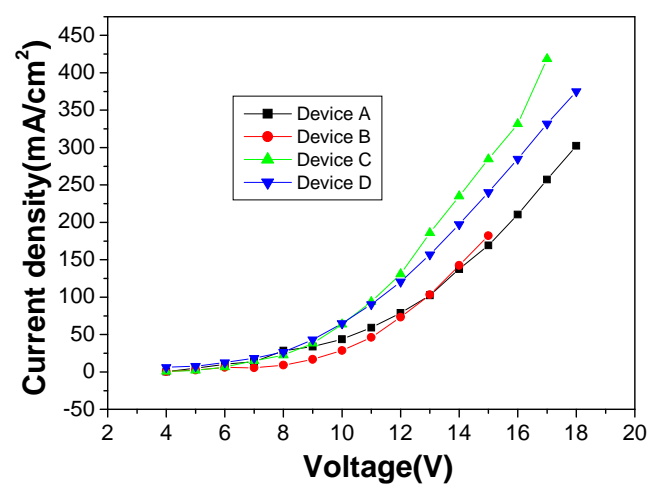

FIGURE II. (A) CURRENT DENSITY -VOLTAGE CHARACTERISTICS OF DEVICES A-D

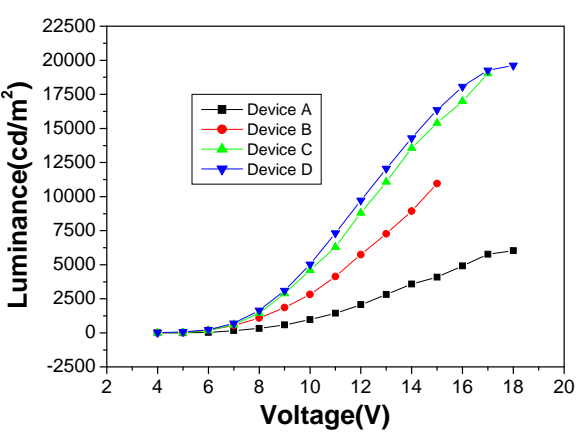

FIGURE II. (B) THE LUMINANCE- VOLTAGE CHARACTERISTICS OF DEVICES A-D 

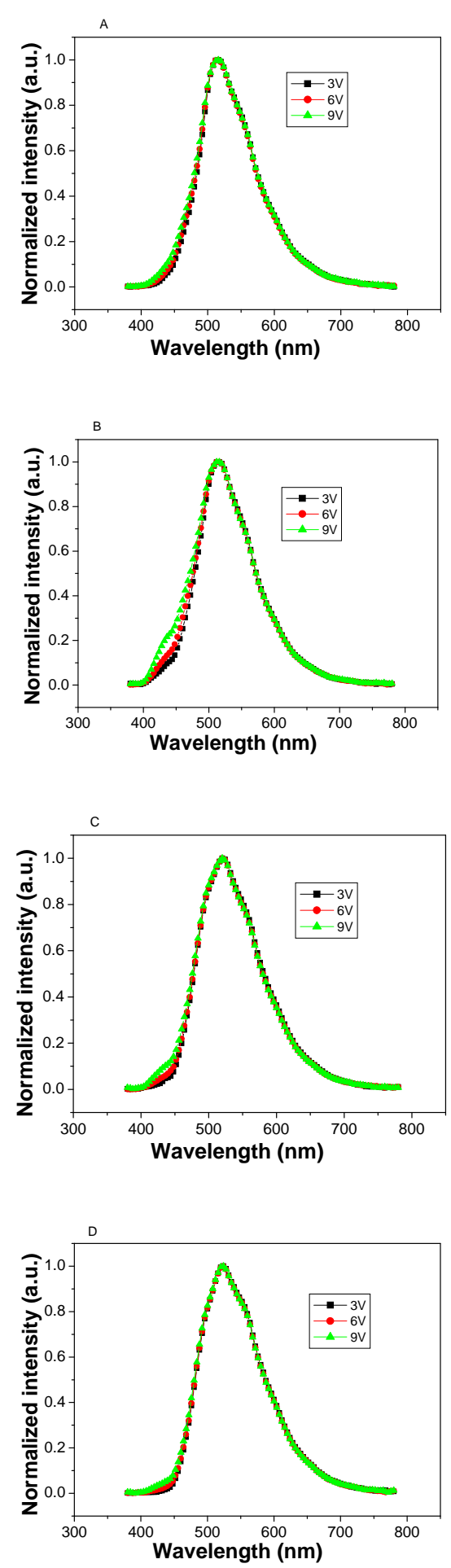

FIGURE III. (A) NORMALIZED EL INTENSITY OF THE DIFFERENT DEVICES A-D AT DIFFERENT VOLTAGE

Figure III (A) shows the electroluminescence spectra of device A-D with varying voltages. In all the devices, green electroluminescence (EL) was observed originating from the electrically excited emissive layer of Irppy3. The normalized
EL spectra of the devices shows main emission peak at $516 \mathrm{~nm}$ originating from Irppy3. The spectral variation was found to be different structure of the devices due to the BAlq as electron transport layer can better excitons blocking noticeably affected the performances of the OLEDs fabricated. Figure 3(b) shows CIE coordinates of devices A-D at different voltage. The CIE coordinates were location in green region and essentially voltage-independent.

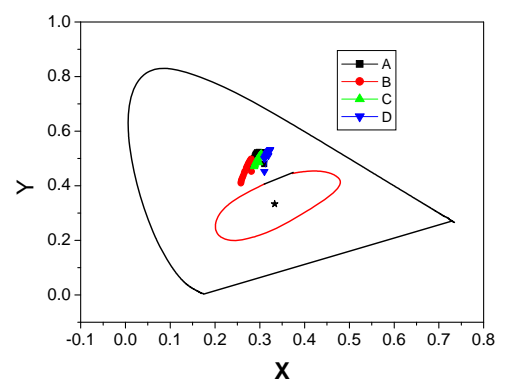

FIGURE III. (B) CIE COORDINATES OF DEVICES A-D AT DIFFERENT VOLTAGE

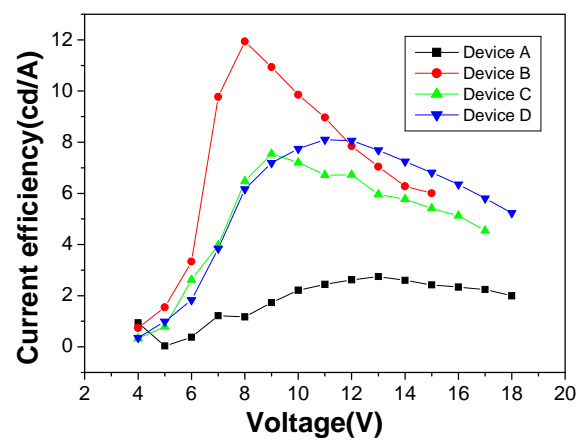

FIGURE IV. (A) THE CURRENT EFFICIENCY- VOLTAGE CHARACTERISTICS OF DEVICES A-D

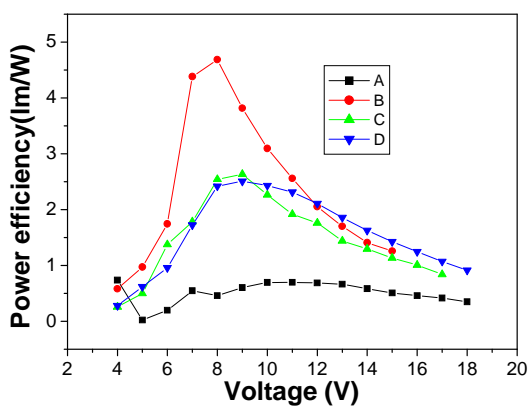

FIGURE IV. (B) THE POWER EFFICIENCY- VOLTAGE CHARACTERISTICS OF DEVICES A-D

Figure 4(a) and figure (b) show the current efficiencyvoltage and the power efficiency-voltage of the devices. It is obvious that the device B with CBP: Alq (6:4) as co-doped host layer has the highest current efficiency $(11.94 \mathrm{~cd} / \mathrm{A})$ that is about 4.3 times higher than that of the device with CBP: Alq 
(8:2) $(2.75 \mathrm{~cd} / \mathrm{A})$. The highest power efficiency $(4.69 \mathrm{~lm} / \mathrm{W})$ that is about 6.7 times higher than that of the device with CBP: Alq $(8: 2)(0.70 \mathrm{~lm} / \mathrm{W})$. Table 1 lists the performance of the devices. We can see that the device B with CBP: Alq (6:4) shows the maximum current efficiency and maximum power efficiency. It indicates that the holes and electrons are more balanced in the OLED with an optimum co-doped host layer of around 6:4 than in another three devices. Our results suggest that the optimum ratio of co-doped host layer improve the balance of hole and electron resulting in the improvement of the efficiency. Usually, the mobility of electrons is much lower than holes in common organic materials. This gives rise to an accumulation of excess that of holes at the EML interface. Either increasing the number of electrons or decreasing the number of holes may improve the balance. Obviously, the presence of CPB: Alq can decrease the number of holes, while the number of electrons can be confined in emitting layer. Such a structure helps to increase the energy transfer from host to guest resulting in an improved current efficiency.

The phenomena observed experimentally are consistent with such a speculation, which can be discussed by follows. The luminous efficiency was improved by co-doped host layer method. The host of the emitter layer was composed of CBP and Alq dopant. The Alq dopant did not emit in the emitter layer because their band gaps were wider than that of $\operatorname{Ir}(\mathrm{ppy}) 3$, and their HOMO and LUMO levels were outside those of $\operatorname{Ir}($ ppy)3. The role of the Alq dopant layer was to supply electrons to the emitting layer for the carrier recombination process and to obstruct the passage of the useless holes through to the cathode. In order to obtain high luminous efficiency, the passage of the useless holes should be prevented effectively.

\section{CONCLUSION}

In summary, to achieve the high efficient OLEDs, both carriers and excitons must be well confined within the emissive layer. The approach discussed here is to efficiently force carrier recombination and exciton confinement in the EML by the use of co-doped host layer method. By carefully controlling the ratio of co-doped host materials, it may be possible to balanced holes and electrons at EML/ETL interfaces. The result is a simplified structure for high efficiency devices, we have demonstrated a high-efficiency OLEDs with CBP: Alq as codoped host layer. The highest luminous efficiency of the device with 6:4 of CBP: Alq achieved $11.9 \mathrm{~cd} / \mathrm{A}$, which is nearly 4.3 times that of the device with 8:2 of CBP: Alq, and the power efficiency achieved $4.69 \mathrm{~lm} / \mathrm{W}$, which is nearly 6.7 times that of the device with 8:2 of CBP: Alq.

\section{ACKNOWLEDGMENTS}

This work was supported by the major project of science and technology office of Fujian Province of China (No.2014H0042), the Natural Science Foundation of Fujian Province of China (No. 2015J01664), the project of Science and Technology Research of Quanzhou, Fujian Provice of China (No. 2014Z137), and The 2016 annual national or ministries of the Quanzhou Normal University Prepare Research Foundation Project (No.2016YYKJ21).

\section{REFERENCES}

[1] Murawski, C., Leo, K. \& Gather, M. C. Efficiency Roll-Off in Organic Light-Emitting Diodes. Adv. Mater. 25(2013), 6801-6827.

[2] Bing Chen, Yanhu Li, Yueying Chu, Anmin Zheng, Jiwen Feng, Zhitian Liu, Hongbin Wu, Wei Yang, Highly efficient single-layer organic lightemitting devices using cationic iridium complex as host, Organic Electronics, 14(2013) 744 .

[3] Dong-Hyun Lee, Jong Hyeok Park, Heeyeop Chae, Sung M. Cho, Enhanced carrier balance by organic salt doping in single-layer polymer light-emitting devices, Organic Electronics, 10 (2009)1345.

[4] Chih-Hung Hsiao, Shun-Wei Liu, Chin-Ti Chen, Jiun-Haw Lee, Emitting layer thickness dependence of color stability in phosphorescent organic light-emitting devices, Organic Electronics, 11(2010)1500.

[5] Sang-Hyun Eom, Ying Zheng, Edward Wrzesniewski, Jaewon Lee, Neetu Chopra, Franky So, Jiangeng Xue, Effect of electron injection and transport materials on efficiency of deep-blue phosphorescent organic light-emitting devices, Organic Electronics, 10(2009)686.

[6] Shixiong Liang, Zhaoxin Wu, Xuanke Zhao, Dawei Wang, Xun Hou, Zhijian Chen, Qihuang Gong, Investigation on the escaped and trapped emission in organic light-emitting devices, Optics Communications, 285(2012)1625.

[7] Yu Bai, Jing Feng, Yue-Feng Liu, Jun-Feng Song, Janne Simonen, Yu Jin, Qi-Dai Chen, Jian Zi, Hong-Bo Sun, Outcoupling of trapped optical modes in organic light-emitting devices with one-step fabricated periodic corrugation by laser ablation, Organic Electronics, 12(2011)1927.

[8] V. E. Choong, S. Shi, J. Curless, C. L. Shieh, H. C. Lee, J. Shen, J. Yang, Organic light-emitting diodes with a bipolar transport layer, Appl.Phys.Lett.75(1999)172.

[9] A. B. Chwang, R. C. Kwong, J. J. Brown, Graded mixed-layer organic light-emitting devices, Appl. Phys. Lett. 80(2002)725.

[10] D. Ma, C. S. Lee, S. T. Lee, L. S. Hung, Improved efficiency by a graded emissive region in organic light-emitting diodes, Appl.Phys.Lett.80(2002)3641.

[11] Y. Shao, Y. Yang, Naturally formed graded junction for organic lightemitting diodes, Appl. Phys. Lett. 83(2003)2453.

[12] N. C. Erickson, R. J. Holmes, Highly efficient, single-layer organic light-emitting devices based on a graded-composition emissive layer, Appl.Phys.Lett.97(2010)083308.

[13] C. H. Hsiao, Y. H. Chen, T. C. Lin, C. C. Hsiao, J. H. Lee, Recombination zone in mixed-host organic light-emitting devices, Appl.Phys.Lett.89(2006)163511.

[14] C. W. Chen, T. Y. Cho, C. C. Wu, H. L. Yu, T. Y. Luh, Fuzzy-junction organic light-emitting devices, Appl.Phys. Lett.81(2002)1570. 